\title{
Multiple solutions for impulsive higher order functional differential equations
}

M. Benchohra, J. Henderson, S. K. Ntouyas, and A. Ouahab 


\title{
MULTIPLE SOLUTIONS FOR IMPULSIVE HIGHER ORDER FUNCTIONAL DIFFERENTIAL EQUATIONS
}

\author{
M. BENCHOHRA, J. HENDERSON, S. K. NTOUYAS, AND A. OUAHAB
}

Received 17 May, 2006

\begin{abstract}
The Leggett-Williams fixed point theorem is applied to obtain triple positive solutions for impulsive $n$th order functional differential equations.
\end{abstract}

2000 Mathematics Subject Classification: 34A37, 34K25

Keywords: impulsive functional differential equations, multiple solutions, concave functional

\section{INTRODUCTION}

In this paper, we are concerned with the existence of three nonnegative solutions for initial value problems for $n$th order functional differential equations with impulsive effects

$$
\begin{gathered}
y^{(n)}(t)=f\left(t, y_{t}\right), \quad t \in J=[0, T], t \neq t_{k}, k=1, \ldots, m, \\
\left.\Delta y^{(i-1)}\right|_{t=t_{k}}=I_{k}^{i}\left(y\left(t_{k}^{-}\right)\right), \quad i=1, \ldots, n, k=1, \ldots, m, \\
y(t)=\phi(t), \quad t \in[-r, 0], \\
y^{(i-1)}(0)=\eta_{i}, \quad i=2, \ldots, n,
\end{gathered}
$$

where $f: J \times D \rightarrow \mathbb{R}$ is a given function, $D=\left\{\psi:[-r, 0] \rightarrow \mathbb{R}_{+} \mid \psi\right.$ is continuous everywhere except for a finite number of points $s$ at which $\psi(s)$ and the right limit $\psi\left(s^{+}\right)$exist and $\left.\psi\left(s^{-}\right)=\psi(s)\right\}, \phi \in D, 0<r<\infty, \eta_{i} \in \mathbb{R}, 0=t_{0}<t_{1}<\cdots<t_{m}<$ $t_{m+1}=T, I_{k}^{i} \in C\left(\mathbb{R}, \mathbb{R}_{+}\right)(i=1, \ldots, n, k=1,2, \ldots, m),\left.\Delta y\right|_{t=t_{k}}=y\left(t_{k}^{+}\right)-y\left(t_{k}^{-}\right)$, and $\mathbb{R}$ is a real Banach space with the norm $|\cdot|$.

For any function $y$ defined on $[-r, T]$ and any $t \in J$, we denote by $y_{t}$ the function defined by

$$
y_{t}(\theta)=y(t+\theta), \quad \theta \in[-r, 0] .
$$

Impulsive differential equations can be used to model and describe real processes and phenomena studied in physics, chemical technology, population dynamics, biotechnology and economics. That is why, in recent years, they have become an object of investigation. We refer to the monographs of Bainov and Simeonov [5], Benchohra, Henderson and Ntouyas [6], Lakshmikantham et al [14], and Samoilenko 
and Perestyuk [16] where numerous properties of their solutions are studied, and a detailed bibliography is given.

Recently extensions to functional differential equations with impulsive effects have been done by Benchohra et al. [7], Dong [9], and Franco et al. [11].

The existence of multiple solutions for boundary value problems for impulsive differential equations was studied by Agarwal and O'Regan [1], Erbe et al. [10], and Guo and Liu [13]. Notice that when the impulses are absent (i.e., $I_{k}=0$, $k=1, \ldots, m)$ the existence of three solutions and multiple solutions for ordinary differential equations was studied in [2-4].

The main results here make use of the Leggett-Williams fixed point theorem [15] in obtaining multiple positive solutions for the problem (1.1)-(1.4). This paper can be viewed as a generalization of the recent paper [8].

\section{PReliminaries}

In this section, we provide notations, definitions, and preliminary facts which are used throughout the paper.

By $C(J, \mathbb{R})$ we denote the Banach space of all continuous functions from $J$ into $\mathbb{R}$ with the norm

$$
\|y\|_{J}:=\sup \{|y(t)|: t \in J\} .
$$

$L^{1}(J, \mathbb{R})$ denotes the Banach space of measurable functions $y: J \rightarrow \mathbb{R}$ which are Lebesgue integrable, with

$$
\|y\|_{L^{1}}=\int_{0}^{T}|y(t)| d t .
$$

Let $(E,\|\cdot\|)$ be a Banach space and $C$ be a cone in $E$. By a concave nonnegative continuous functional $\psi$ on $C$ we mean a continuous mapping $\psi: C \rightarrow[0, \infty]$ with

$$
\psi(\lambda x+(1-\lambda) y) \geq \lambda \psi(x)+(1-\lambda) \psi(y) \text { for all } x, y \in C \text { and } \lambda \in[0,1] .
$$

For $k=0, \ldots, m$, let $J_{k}=\left(t_{k}, t_{k+1}\right]$ and for $y:[-r, T] \rightarrow \mathbb{R}$, define $y_{k}$ to be the restriction of $y$ to $J_{k}$. In order to define the solution of (1.1)-(1.4), we shall consider the space

$$
\Omega=\left\{y:[-r, T] \rightarrow \mathbb{R} \mid y_{k} \in C\left(J_{k}, \mathbb{R}\right), k=0, \ldots, m,\right. \text { and }
$$

$$
\text { there exists } \left.y\left(t_{k}^{+}\right), k=1, \ldots, m\right\} \text {, }
$$

which is a Banach space with the norm

$$
\|y\|_{\Omega}=\max \left\{\left\|y_{k}\right\|_{J_{k}}: k=0, \ldots, m\right\} .
$$

Definition 1. A map $f: J \times D \rightarrow \mathbb{R}$ is said to be $L^{1}$-Carathéodory if

(i) $t \mapsto f(t, u)$ is measurable for each $u \in D$;

(ii) $u \mapsto f(t, u)$ is continuous for almost all $t \in J$; 
(iii) for each $q>0$, there exists $h_{q} \in L^{1}\left(J, \mathbb{R}_{+}\right)$such that

$$
|f(t, u)| \leq h_{q}(t) \text { for all }\|u\| \leq q \text { and for almost all } t \in J .
$$

Our consideration is based on the following fixed point theorem by Leggett and Williams [15] (see also Guo and Lakshmikantham [12]).

Theorem 2.1. Let $E$ be a Banach space, $C \subset E$ a cone of $E$ and $R>0$ a constant. Let $C_{R}=\{y \in C:\|y\|<R\}$. Suppose a concave nonnegative continuous functional $\psi$ exists on the cone $C$ with $\psi(y) \leq\|y\|$ for $y \in \bar{C}_{R}$, and let $N: \bar{C}_{R} \rightarrow \bar{C}_{R}$ be a completely continuous operator. Assume there are numbers $\varrho, L$ and $K$ with $0<\varrho<L<K \leq R$ such that

(A1) $\{y \in C(\psi, L, K): \psi(y)>L\} \neq \varnothing$ and $\psi(N(y))>L$ for all $y \in C(\psi, L, K)$;

(A2) $\|N(y)\|<\varrho$ for all $y \in \bar{C}_{\varrho}$;

(A3) $\psi(N(y))>L$ for all $y \in C(\psi, L, R)$ with $\|N(y)\|>K$, where $C(\psi, L, K)=$ $\{y \in C: \psi(y) \geq L$ and $\|y\| \leq K\}$.

Then $N$ has at least three fixed points $y_{1}, y_{2}, y_{3}$ in $\bar{C}_{R}$. Furthermore, we have

$$
y_{1} \in C_{\varrho}, \quad y_{2} \in\{y \in C(\psi, L, R): \psi(y)>L\},
$$

and

$$
y_{3} \in \bar{C}_{R} \backslash\left\{C(\psi, L, R) \cup \bar{C}_{\varrho}\right\}
$$

\section{TRIPLE SOLUTIONS OF IMPULSIVE FUNCTIONAL DIFFERENTIAL EQUATIONS}

In what follows we will assume that $f$ is an $L^{1}$-Carathéodory function. Let us start by defining what we mean by a solution of problem (1.1)-(1.4).

Definition 2. A function $y \in \Omega$ such that $y \in \Omega \cap A C^{(n-1)}\left(\left(t_{k}, t_{k+1}\right), \mathbb{R}\right)$, for $k=$ $0, \ldots, m$, is said to be a solution of (1.1)-(1.4) if $y$ satisfies the equation $y^{(n-1)}(t)=$ $f\left(t, y_{t}\right)$ a.e. on $J \backslash\left\{t_{1}, \ldots, t_{m}\right\}$, the conditions $\left.\Delta y^{(i-1)}\right|_{t=t_{k}}=I_{k}^{i}\left(y\left(t_{k}^{-}\right)\right), i=$ $1, \ldots, n, k=1, \ldots, m, y(t)=\phi(t), t \in[-r, 0]$, and $y^{(i-1)}(0)=\eta_{i}, i=2, \ldots, n$.

Here, $A C^{(n-1)}\left(\left(t_{k}, t_{k+1}\right), \mathbb{R}\right)=\left\{y:\left(t_{k}, t_{k+1}\right) \rightarrow \mathbb{R} \mid y^{(i)}\right.$ is absolutely continuous, $i=0, \ldots, n-1\}$.

In our application of Theorem 2.1 to obtain triple positive solutions of (1.1)-(1.4), we will draw upon the following list of hypotheses.

(H1) There exist constants $c_{k}^{i}, i=1, \ldots, n, k=1, \ldots, m$, such that

$$
I_{k}^{i}(y) \leq c_{k}^{i}, \quad i=1, \ldots, n, k=1, \ldots, m, \text { for each } y \in \mathbb{R} ;
$$

(H2) There exists a function $h \in L^{1}\left(J, \mathbb{R}_{+}\right)$and $\varrho>0$ and $0<M<1$ such that

$$
f(t, u) \leq M h(t) \text { for a.e. } t \in J \text { and each } u \in D
$$


and

$$
\begin{aligned}
\|\phi\|+\sum_{i=2}^{n}\left|\eta_{i}\right| \frac{T^{i-1}}{(i-1) !} & +M \int_{0}^{T} \frac{(T-s)^{n-1}}{(n-1) !} h(s) d s \\
& +\sum_{k=1}^{m} \sum_{i=1}^{n} \frac{\left(T-t_{k}\right)^{i-1}}{(i-1) !} c_{k}^{i}<\varrho
\end{aligned}
$$

(H3) There exist $L>\varrho$ and $M \leq M_{1}<1$ and an interval $[a, b] \subset(0, T)$ such that, for each $y \in \Omega$ with $\min _{t \in[a, b]} y(t) \geq L$,

$$
\begin{aligned}
& \begin{aligned}
\min _{t \in[a, b]}\left(\phi(0)+\sum_{i=2}^{n} \eta_{i} \frac{t^{i-1}}{(i-1) !}\right. & +\int_{0}^{t} \frac{(t-s)^{n-1}}{(n-1) !} f\left(s, y_{s}\right) d s \\
& \left.+\sum_{0<t_{k}<t} \sum_{i=1}^{n} \frac{\left(t-t_{k}\right)^{i-1}}{(i-1) !} I_{k}^{i}\left(y\left(t_{k}^{-}\right)\right)\right) \\
\geq M_{1}\left(\phi(0)+\sum_{i=2}^{n} \eta_{i} \frac{T^{i-1}}{(i-1) !}\right. & +\int_{0}^{T} \frac{T^{n-1}}{(n-1) !} f\left(s, y_{s}\right) d s \\
& \left.+\sum_{k=1}^{m} \sum_{i=1}^{n} \frac{\left(T-t_{k}\right)^{i-1}}{(i-1) !} I_{k}^{i}\left(y\left(t_{k}^{-}\right)\right)\right)
\end{aligned} \\
& >L ;
\end{aligned}
$$

(H4) There exist $R>L$ and $M_{1} \leq M_{2}<1$ such that

$$
\begin{aligned}
\|\phi\|+\sum_{i=2}^{n} \eta_{i} \frac{T^{i-1}}{(i-1) !} & +M_{2} \int_{0}^{T} \frac{(T-s)^{n-1}}{(n-1) !} h(s) d s \\
& +\sum_{k=1}^{m} \sum_{i=1}^{n} \frac{\left(T-t_{k}\right)^{i-1}}{(i-1) !} c_{k}^{i} \leq R .
\end{aligned}
$$

Theorem 3.1. Suppose that hypotheses $(\mathrm{H} 1)-(\mathrm{H} 4)$ are satisfied. Then problem (1.1)-(1.4) has three nonnegative solutions $y_{1}, y_{2}$, and $y_{3}$ with

$$
\left\|y_{1}\right\|_{\Omega}<\varrho, \quad y_{2}(t)>L \quad \text { for } t \in[0, T],
$$

and

$$
\left\|y_{3}\right\|_{\Omega}>\varrho \quad \text { with } \min _{t \in[a, b]} y_{3}(t)<L
$$


Proof. In making application of Theorem 2.1, we naturally transform problem (1.1)-(1.4) into a fixed point problem. Define an operator $N: \Omega \rightarrow \Omega$ by

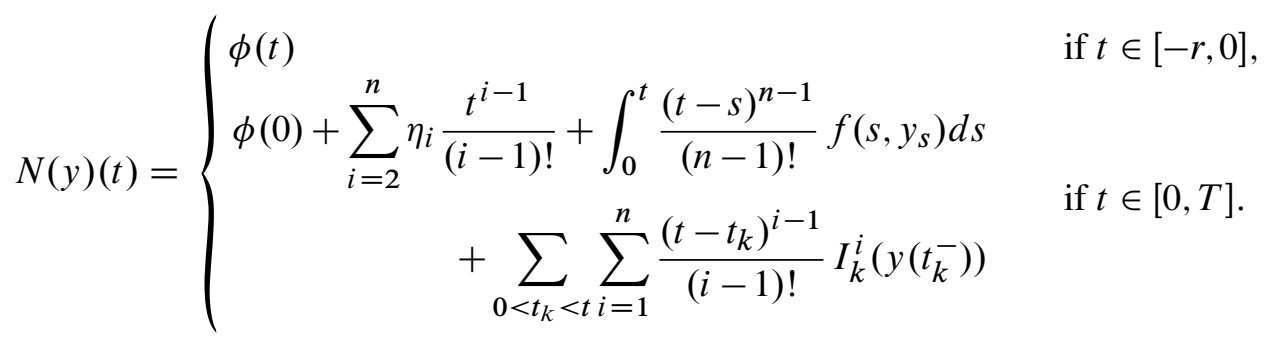

It is fairly standard that $N$ is completely continuous. Rather than repeat the usual complete continuity arguments, we refer the reader to $[7,8]$ for details. We content ourselves in verifying that the other hypotheses of Theorem 2.1 are satisfied. Let

$$
C=\{y \in \Omega: y(t) \geq 0 \text { for } t \in[-r, T]\}
$$

be a cone in $\Omega$. Since $f$ and $I_{k}^{i}$ are all positive functions, it is immediate that $N(C) \subset$ $C$, and moreover, $\left.N\right|_{\bar{C}_{R}}$ is completely continuous. In fact, if $y \in \bar{C}_{R}$, then by (H4), $\|N(y)\|_{\Omega} \leq R$. In particular, $N: \bar{C}_{R} \rightarrow \bar{C}_{R}$ is completely continuous.

Next, let $\psi: C \rightarrow[0, \infty)$ be defined by

$$
\psi(y)=\min _{t \in[a, b]} y(t)
$$

Then, it is clear that $\psi$ is a nonnegative concave continuous functional and

$$
\psi(y) \leq\|y\|_{\Omega} \quad \text { for } y \in \bar{C}_{R} .
$$

Now it remains to show that the hypotheses (A1) and (A2) of Theorem 2.1 are satisfied. First, for $y \in \bar{C}_{\varrho}$, we have from (H1) and (H2),

$$
\begin{aligned}
&|N(y)(t)| \leq|\phi(0)|+\sum_{i=2}^{n}\left|\eta_{i}\right| \frac{T^{i-1}}{(i-1) !}+\int_{0}^{T} \frac{(T-s)^{n-1}}{(n-1) !} f\left(s, y_{s}\right) d s \\
&+\sum_{0<t_{k}<t} \sum_{i=1}^{n}+\frac{\left(T-t_{k}\right)^{i-1}}{(i-1) !} I_{k}\left(y\left(t_{k}^{-}\right)\right) \\
& \leq\|\phi\|+\sum_{i=2}^{n}\left|\eta_{i}\right| \frac{T^{i-1}}{(i-1) !}+M \int_{0}^{T} \frac{(T-s)^{n-1}}{(n-1) !} h(s) d s \\
&+\sum_{k=1}^{m} \sum_{i=1}^{n} \frac{\left(T-t_{k}\right)^{i-1}}{(i-1) !} c_{k}^{i} \\
&<\varrho .
\end{aligned}
$$

As a consequence, (A2) of Theorem 2.1 holds. Next, let $K$ be such that $\frac{L}{M} \leq K \leq R$ and let $z(t)=\frac{1}{2}(L+K)$ for $t \in[-r, T]$. By the definition of $C(\psi, L, K)$, the function 
$z$ belongs to $C(\psi, L, K)$. Moreover, $\psi(z)=\frac{1}{2}(L+K)>L$, so that

$$
\{y \in C(\psi, L, K): \psi(y)>L\} \neq \varnothing .
$$

So, if we choose $y \in C(\psi, L, K)$, then from (H3), we have

$$
\begin{aligned}
\psi(N(y))= & \min _{t \in[a, b]}\left(\phi(0)+\sum_{i=2}^{n} \eta_{i} \frac{t^{i-1}}{(i-1) !}+\sum_{0<t_{k}<t} \sum_{i=1}^{n} \frac{\left(t-t_{k}\right)^{i-1}}{(i-1) !} I_{k}^{i}\left(y\left(t_{k}\right)\right)\right. \\
& \left.+\int_{0}^{t} \frac{(t-s)^{n-1}}{(n-1) !} f\left(s, y_{s}\right) d s\right) \\
\geq & M_{1}\left(\phi(0)+\sum_{i=2}^{n} \eta_{i} \frac{T^{i-1}}{(i-1) !}+\int_{0}^{T} \frac{T^{n-1}}{(n-1) !} f\left(s, y_{s}\right) d s\right. \\
& \left.+\sum_{k=1}^{m} \sum_{i=1}^{n} \frac{\left(T-t_{k}\right)^{i-1}}{(i-1) !} I_{k}^{i}\left(y\left(t_{k}^{-}\right)\right)\right) \\
> & L .
\end{aligned}
$$

As a consequence, (A1) of Theorem 2.1 is fulfilled.

Finally, to see that (A3) of Theorem 2.1 holds, let $y \in C(\psi, L, R)$ with $\|N(y)\|_{\Omega}>$ $K$. Again from (H3), we have

$$
\begin{aligned}
\psi(N(y))= & \min _{t \in[a, b]}\left(\phi(0)+\sum_{i=2}^{n} \eta_{i} \frac{t^{i-1}}{(i-1) !}+\sum_{0<t_{k}<t} \sum_{i=1}^{n} \frac{\left(t-t_{k}\right)^{i-1}}{(i-1) !} I_{k}^{i}\left(y\left(t_{k}\right)\right)\right. \\
& \left.+\int_{0}^{t} \frac{(t-s)^{n-1}}{(n-1) !} f\left(s, y_{s}\right) d s\right) \\
\geq & M_{1}\left(\phi(0)+\sum_{i=2}^{n} \eta_{i} \frac{T^{i-1}}{(i-1) !}+\int_{0}^{T} \frac{T^{n-1}}{(n-1) !} f\left(s, y_{s}\right) d s\right. \\
& \left.+\sum_{k=1}^{m} \sum_{i=1}^{n} \frac{\left(T-t_{k}\right)^{i-1}}{(i-1) !} I_{k}^{i}\left(y\left(t_{k}^{-}\right)\right)\right) \\
\geq & M_{1}\|N(y)\|_{\Omega}>M_{1} K \geq L .
\end{aligned}
$$

Thus, Theorem 2.1 implies that $N$ has at least three fixed points $y_{1}, y_{2}$, and $y_{3}$ which are positive solutions of problem (1.1)-(1.4). Furthermore, we have $y_{1} \in C_{\varrho}$,

$$
y_{2} \in\{y \in C(\psi, L, R): \psi(y)>L\},
$$

and $y_{3} \in C_{R} \backslash\left(C(\psi, L, R) \cup C_{\varrho}\right)$.The proof is complete.

\section{REFERENCES}

[1] R. P. Agarwal and D. O'Regan, "Multiple nonnegative solutions for second order impulsive differential equations," Appl. Math. Comput., vol. 114, no. 1, pp. 51-59, 2000. 
[2] R. P. Agarwal and D. O'Regan, "Existence of three solutions to integral and discrete equations via the Leggett Williams fixed point theorem," Rocky Mountain J. Math., vol. 31, no. 1, pp. 23-35, 2001.

[3] R. P. Agarwal, D. O’Regan, and P. J. Y. Wong, Positive solutions of differential, difference and integral equations. Dordrecht: Kluwer Academic Publishers, 1999.

[4] D. Anderson, R. Avery, and A. Peterson, "Three positive solutions to a discrete focal boundary value problem," J. Comput. Appl. Math., vol. 88, no. 1, pp. 103-118, 1998, positive solutions of nonlinear problems.

[5] D. D. Bainnov and P. S. Simeonov, Systems with impulse effect, ser. Ellis Horwood Series: Mathematics and its Applications. Chichester: Ellis Horwood Ltd., 1989, stability, theory and applications.

[6] M. Benchohra, J. Henderson, and S. Ntouyas, Impulsive differential equations and inclusions, ser. Contemporary Mathematics and Its Applications. Hindawi Publishing Corporation, New York, 2006, vol. 2.

[7] M. Benchohra, J. Henderson, and S. K. Ntouyas, "An existence result for first-order impulsive functional differential equations in Banach spaces," Comput. Math. Appl., vol. 42, no. 10-11, pp. 1303-1310, 2001.

[8] M. Benchohra, A. Ouahab, J. Henderson, and S. K. Ntouyas, "A note on multiple solutions for impulsive functional differential equations," Comm. Appl. Nonlinear Anal., vol. 12, no. 3, pp. 61-70, 2005.

[9] Y. Dong, "Periodic boundary value problems for functional-differential equations with impulses," J. Math. Anal. Appl., vol. 210, no. 1, pp. 170-181, 1997.

[10] L. H. Erbe, S. C. Hu, and H. Wang, "Multiple positive solutions of some boundary value problems," J. Math. Anal. Appl., vol. 184, no. 3, pp. 640-648, 1994.

[11] D. Franco, E. Liz, J. J. Nieto, and Y. V. Rogovchenko, "A contribution to the study of functional differential equations with impulses," Math. Nachr., vol. 218, pp. 49-60, 2000.

[12] D. J. Guo and V. Lakshmikantham, Nonlinear problems in abstract cones, ser. Notes and Reports in Mathematics in Science and Engineering. Boston, MA: Academic Press Inc., 1988, vol. 5.

[13] D. J. Guo and X. Liu, "Multiple positive solutions of boundary-value problems for impulsive differential equations," Nonlinear Anal., vol. 25, no. 4, pp. 327-337, 1995.

[14] V. Lakshmikantham, D. D. Baŭnov, and P. S. Simeonov, Theory of impulsive differential equations, ser. Series in Modern Applied Mathematics. Teaneck, NJ: World Scientific Publishing Co. Inc., 1989, vol. 6.

[15] R. W. Leggett and L. R. Williams, "Multiple positive fixed points of nonlinear operators on ordered Banach spaces," Indiana Univ. Math. J., vol. 28, no. 4, pp. 673-688, 1979.

[16] A. M. Samoŭlenko and N. A. Perestyuk, Impulsive differential equations, ser. World Scientific Series on Nonlinear Science. Series A: Monographs and Treatises. River Edge, NJ: World Scientific Publishing Co. Inc., 1995, vol. 14.

\section{Authors' addresses}

\section{Benchohra}

Laboratoire de Mathématiques, Université de Sidi Bel Abbès, BP 89, 22000 Sidi Bel Abbès, Algérie

E-mail address: benchohra@univ-sba.dz

\section{J. Henderson}

Baylor University, Department of Mathematics, Waco, Texas 76798-7328 USA

E-mail address: Johnny_Henderson@baylor.ed 
S. K. Ntouyas

Department of Mathematics, University of Ioannina, 45110 Ioannina, Greece

E-mail address: sntouyasecc.uoi.gr

A. Ouahab

Laboratoire de Mathématiques, Université de Sidi Bel Abbès, BP 89, 22000 Sidi Bel Abbès, Algérie

E-mail address: agh_ouahab@yahoo.fr 